\title{
Indicadores do Serviço de Atenção Farmacêutica (SAF) da Universidade do Sul de Santa Catarina
}

\author{
Indicators of the pharmaceutical care service \\ at the University of Southern Santa Catarina
}

Graziela Modolon Alano ${ }^{1}$ Taís dos Santos Corrêa ${ }^{1}$ Dayani Galato ${ }^{1}$
${ }^{1}$ Curso de Farmácia, Departamento de Ciências Biológicas e da Saúde e de Ciências Sociais Aplicadas, Universidade do Sul de Santa Catarina. Avenida José Acácio Moreira787, Dehon. 88704-900 Tubarão SC. graziela.alano@unisul.br
Abstract Pharmaceutical Care has represented a new clinical practice for pharmacists. This study aims to show the indicators of the Pharmaceutical Care Service at the University of Southern Santa Catarina. Documentary analysis of pharmaceutical records of 58 patients was conducted between September 2007 and March 2008 to identify the indicators. Most patients were women (77.6\%), with a mean age of 54 years. Average number of health problems per patient was 4.6 in the first visit and 4.3 in the last one. The most widely used drug classes were cardiovascular (30.2\%) and nervous system (27.5\%). On average, 2.7 drug-related problems per patient were detected, the most common being those related to effectiveness and safety. Of the problems identified, $82.2 \%$ were classified as preventable and $63.7 \%$ as manifested. Pharmaceutical interventions registered $79.0 \%$ of acceptance, of which $78.9 \%$ had positive results. On average, there were $2.6 \mathrm{pa}$ tient-related needs, especially regarding questions about drug therapy (30.4\%), which were positively answered in $84.8 \%$ of cases. These indicators show that the pharmaceutical care service is very active in promoting health education.

Key words Pharmaceutical care, Patient-related needs, Drug-related problems, Patient-oriented services
Resumo A Atenção Farmacêutica tem se constituído numa nova prática clínica para o farmacêutico. O estudo tem por objetivo apresentar os indicadores do Serviço de Atenção Farmacêutica da Universidade do Sul de Santa Catarina. Foi realizada a análise documental dos prontuários farmacêuticos de 58 pacientes entre setembro de 2007 a março de 2008. A maioria dos pacientes eram mulheres $(77,6 \%)$ com idade média de 54 anos. Identificou-se no primeiro encontro a média de 4,6 problemas de saúde por paciente e no último 4,3. As classes farmacológicas mais prevalentes foram a cardiovascular (30,2\%) e no sistema nervoso (27,5\%). Detectou-se média de 2,7 problemas relacionados com medicamentos por paciente, sendo os mais comuns aqueles relacionados à efetividade e à segurança. Dos problemas identificados $82,2 \%$ foram classificados como evitáveis e 63,7\% como manifestados. Das intervenções farmacêuticas registradas soube-se da aceitação de 79,0\% com 78,9\% de resultados positivos. Encontrou-se média de 2,6 necessidades relacionadas ao paciente sendo, principalmente, dúvidas quanto à terapia farmacológica (30,4\%) sendo em $84,8 \%$ dos casos supridas.

Palavras-chave Atenção farmacêutica, Necessidade relacionada ao paciente (NRP), Problema relacionado ao medicamento (PRM), Serviço voltado ao paciente 


\section{Introdução}

O uso de terapia farmacológica não está isenta de problemas. Os tratamentos com medicamentos podem gerar diversos efeitos indesejados e fica evidente que a segurança dos medicamentos é uma questão bastante estudada. Além disso, há problemas advindos do uso dos medicamentos relativos à efetividade dos tratamentos, pois, em diversos casos, não se consegue alcançar o objetivo terapêutico para os quais foram prescritos, seja por causas relacionadas ao paciente, aos profissionais de saúde ou ao próprio medicamento ${ }^{1}$.

Ao conjunto de todas essas experiências indesejáveis denomina-se de Problema Relacionado ao Medicamento (PRM) definido pelo Terceiro Consenso de Granada ${ }^{2}$ como aquelas situações em que o processo de uso de medicamentos causa ou pode causar a aparição de um resultado negativo associado à medicação. Este pode ser relacionado à segurança, à efetividade ou à necessidade da terapia farmacológica. Portanto, o PRM caracteriza-se como um problema de saúde, derivado do tratamento farmacológico que, produzido por diversas causas têm como consequência o não alcance do objetivo terapêutico desejado ou $o$ aparecimento de efeitos indesejáveis ${ }^{3}$.

A participação do farmacêutico na atenção individualizada dos pacientes tem aumentado em diversos ambientes assistenciais (comunitário e hospitalar) e tem mostrado um impacto positivo na melhora da saúde dos pacientes ${ }^{4}$. Esta ação individualizada tem sido denominada Atenção Farmacêutica que, no Brasil, começou a ser implementada desde 2000 e passou a ser divulgada, principalmente, a partir das oficinas desenvolvidas pela Organização Pan-Americana de Saúde junto de outras entidades Nacionais (2001-2002) ${ }^{5}$.

O processo de atenção farmacêutica envolve entrevistas com o paciente tendo por objetivo prevenir, identificar e resolver o PRM. O farmacêutico, então, busca solucionar o PRM identificado por meio de uma intervenção farmacêutica. Entende-se por intervenção farmacêutica a atuação ou a recomendação do farmacêutico, direcionada ao paciente ou ao profissional de saúde, para resolver ou prevenir um ou mais $\mathrm{PRM}^{6}$. Nas intervenções que compreendem interrupção do uso do medicamento, mudança de forma farmacêutica ou princípio ativo, dose e intervalo de administração, o farmacêutico deverá, impreterivelmente, entrar em contato com o prescritor e, assim comunicá-lo da necessidade identificada, solicitando auxílio para a intervenção. Por outro lado, há intervenções como, o in- centivo à mudança de estilo de vida, que podem ser feitas diretamente pelo farmacêutico ${ }^{7}$.

Diversos estudos tentam determinar a prevalência dos problemas relacionados aos medicamentos devido a sua relevância em termos financeiros para o Sistema de Saúde do País, ressaltando que estes podem ser a causa de cerca de $30 \%$ das assistências hospitalares ${ }^{1}$. Segundo Mota $^{8}$, os resultados alcançados por meio de intervenções farmacêuticas podem contribuir para a diminuição dos gastos em internações hospitalares, em prescrições de medicamentos adicionais e em ganho na qualidade de vida dos pacientes. Portanto, dentre as medidas orientadas a reduzir os gastos em farmácia e outros serviços relacionados à saúde do paciente, destacam-se o fortalecimento da atenção farmacêutica e a realização de estudos de avaliação destes serviços.

O serviço de atenção farmacêutica compreende o acompanhamento farmacoterapêutico ${ }^{9}$ do paciente, que deve ser provido de forma contínua, sistematizada e documentada, em colaboração com o próprio e com os profissionais do sistema de saúde, com o fim de alcançar resultados concretos que melhorem a qualidade de vida dele ${ }^{2}$.

O Serviço de Atenção Farmacêutica (SAF) do Curso de Farmácia da Universidade do Sul de Santa Catarina foi criado em março de 2006 a partir da aprovação de um projeto pelo $\mathrm{CNPq} /$ MS e, atualmente, funciona em uma clínica escola multidisciplinar. Suas ações são voltadas para o uso racional de medicamentos, sendo desenvolvidas ações com a comunidade. Estas podem ser de ampla abrangência, como palestras a grupos de pacientes constituídos ${ }^{10}$, ou envolver atendimentos individuais, que têm como foco principal as orientações sobre o uso correto de medicamentos utilizados pelos pacientes.

Dados do projeto de Atenção Farmacêutica de Minnesota, Estados Unidos, indicam que essa prática não somente melhora a evolução real dos pacientes como também se constitui de um investimento economicamente favorável, o que pode refletir em um ganho de qualidade de vida desses pacientes ${ }^{5}$. Desta forma, este trabalho teve o objetivo de identificar os indicadores do SAF de forma a quantificar e qualificar ${ }^{3}$ os PRM identificados, as intervenções farmacêuticas realizadas e as necessidades de atenção à saúde manifestadas pelos pacientes, denominadas, neste estudo, de Necessidades Relacionadas ao Paciente (NRP). A NRP compreende dúvidas ou carências dos pacientes relacionadas ao problema de saúde, à terapia farmacológica ou à necessidade de encaminhamento a outros profissionais de 
saúde percebidas pelo profissional farmacêutico, entre outros.

A NRP é uma necessidade identificada pelo paciente ou pela equipe de saúde, que está relacionada a uma falta de informação, bem como à compreensão ou à internalização de uma informação transmitida. As necessidades não incluem somente as que correspondem a uma classe de medicamentos ou a uma patologia concreta ${ }^{6}$, mas implicam orientações sobre o sistema de saúde, a alimentação, o monitoramento laboratorial, o encaminhamento a outro profissional, as orientações sobre automedicação responsável, sobre formas de tratamento dos problemas de saúde e demais orientações não farmacológicas $^{11}$. Sendo assim, a ação sobre as Necessidades identificadas não é específica dos farmacêuticos. Porém, naquelas que envolvam a terapia farmacológica, o farmacêutico representa o profissional mais habilitado para atuar nesta situação, fornecendo informações, reforçando as já existentes e incentivando a sua implementação.

\section{Métodos}

Realizou-se um estudo transversal por meio da análise documental dos prontuários farmacêuticos de 58 pacientes atendidos pelo Serviço de Atenção Farmacêutica do curso de Farmácia da Unisul.

O levantamento dos dados foi realizado entre setembro de 2007 a março de 2008. Foi realizada a análise individual dos prontuários farmacêuticos dos pacientes atendidos nos primeiros dois anos de funcionamento do SAF que continham as informações coletadas na primeira entrevista com o farmacêutico, bem como dos encontros seguintes, quando ocorridos. Foram levantados os dados epidemiológicos dos pacientes como a idade, o estado civil e a classificação econômica ${ }^{12}$.

Além disso, foram investigados os indicadores do serviço: número total de problemas de saúde e de medicamentos utilizados na data de entrada no serviço e no encontro anterior à data da análise da ficha por meio da classificação farmacológica segundo Anatomic Therapeutic Chemistry $^{13}$, classificação esta proposta pela Organização Mundial da Saúde e amplamente adotada em Estudos de Utilização de Medicamentos. Foi identificado o número de Problemas Relacionados ao Medicamento (PRM), bem como sua classificação em manifestado ou não? Exemplificando, se um paciente apresentar tosse seca com o uso de captopril, o PRM será considerado manifestado. Classificaram-se os PRM ainda, em evitável ou não ${ }^{14}$. Da mesma forma, se o paciente utilizar captopril concomitante às refeições, diminuindo a eficácia do tratamento, este será um PRM evitável por meio da alteração do horário de administração do medicamento. Por fim, adotou-se a classificação do Segundo Consenso de Granada ${ }^{3}$ em problema de necessidade, de efetividade ou de segurança. Foram investigadas as intervenções realizadas para a prevenção ou a resolução do PRM identificado quanto ao tipo de intervenção, à aceitação e ao resultado alcançado. As Necessidades Relacionadas aos Pacientes (NRP) também foram identificadas e categorizadas, além de investigados os tipos de orientações realizadas para atendê-las.

Os dados coletados foram registrados em um banco de dados criado no programa EpiData versão $3.1^{15}$ para posterior análise das informações coletadas no Programa Epi Info versão $3.32^{16}$, determinando o perfil dos pacientes e dos serviços prestados no SAF. Para estudar a associação entre os dados utilizou-se o teste estatístico da análise de variância para comparação entre médias. Adotou-se um nível de significância de 5\% $(\mathrm{p}<0,05)$.

Este trabalho foi norteado pela Resolução 196 do Conselho Nacional de Saúde ${ }^{17}$, sendo aprovado pelo Comitê de Ética da Universidade do Sul de Santa Catarina.

\section{Resultados}

Durante o período de análise de dados, 58 fichas de pacientes assistidos pelo Serviço de Atenção Farmacêutica da Unisul foram analisadas. A maioria dos pacientes pertencia ao sexo feminino, $77,6 \%$, com média de idade de $54,2( \pm 19,48)$ anos, sendo principalmente casados $(67,2 \%)$. Do total, $86,2 \%$ dos pacientes moravam com a família, apenas $32,8 \%$ possuíam planos de saúde e $43,1 \%$ pertenciam à classe $\mathrm{C}$ de acordo com a Classificação $\mathrm{ABEP}^{12}$.

O número de problemas de saúde por paciente variou na entrada ao serviço entre 1 a 10 , com média de 4,6 $( \pm 1,83)$, e até o último encontro analisado entre 0 e 11 problemas e média de $4,3$ ( $\pm 1,86)$, não representando uma diferença significativa $(\mathrm{p}=0,2441)$.

As classes farmacológicas e seus principais representantes utilizados pelos pacientes no início dos acompanhamentos e no último encontro estão apresentados na Tabela 1 . O consumo de 
Tabela 1. Principais classes farmacológicas utilizadas pelos pacientes na entrada e no último encontro realizado no Serviço de Atenção Farmacêutica segundo Anatomic Therapeutic Chemistry ${ }^{11}$.

\begin{tabular}{|c|c|c|c|c|}
\hline \multirow{2}{*}{ Classes farmacológicas } & \multicolumn{2}{|c|}{$\begin{array}{l}\text { Entrada } \\
(n=339)\end{array}$} & \multicolumn{2}{|c|}{$\begin{array}{c}\text { Saída } \\
(\mathrm{n}=347)\end{array}$} \\
\hline & $\mathrm{n}$ & (\%) & $\mathrm{n}$ & $(\%)$ \\
\hline Fármacos que atuam no trato alimentar e no metabolismo & 52 & 15,4 & 57 & 16,4 \\
\hline Fármacos que atuam no sangue e nos órgãos hematopoiéticos & 27 & 8,0 & 30 & 8,7 \\
\hline Fármacos que atuam no sistema cardiovascular & 103 & 30,2 & 107 & 30,8 \\
\hline Fármacos que atuam no sistema geniturinário e hormônios sexuais & 9 & 2,6 & 10 & 2,9 \\
\hline Preparações sistêmicas hormonais, excluindo hormônios sexuais e insulina & 9 & 2,7 & 10 & 2,9 \\
\hline Anti-infecciosos para uso sistêmico & 6 & 1,8 & 4 & 1,2 \\
\hline Fármacos que atuam no sistema músculo-esquelético & 29 & 8,5 & 29 & 8,4 \\
\hline Fármacos que atuam no sistema nervoso & 93 & 27,5 & 89 & 25,6 \\
\hline Fármacos que atuam no sistema respiratório & 11 & 3,3 & 9 & 2,6 \\
\hline Fármacos de uso dermatológico & 0 & 0,0 & 1 & 0,3 \\
\hline Fármacos que atuam nos órgãos sensoriais & 0 & 0,0 & 1 & 0,3 \\
\hline
\end{tabular}

medicamentos na entrada ao serviço variou de 1 a 13 por paciente $(5,8 \pm 2,76)$ e no último encontro variaram de 0 a 13 medicamentos $(6,0 \pm 2,93)$, não demonstrando um aumento significativo do número de medicamentos $(\mathrm{p}=0,7058)$. Ambos os momentos, predominaram medicamentos dos sistemas cardiovascular e nervoso, sendo que estes foram também os medicamentos que mais estiveram relacionados à ocorrência de problema relacionado ao medicamento.

Foram identificados 157 PRM, com média de $2,7( \pm 1,73)$ por paciente. A frequência e a classificação dos PRM são apresentadas na Tabela 2 . Foram observados com maior frequência entre os pacientes atendidos pelo SAF os PRM 4 e 5, os quais relacionam-se a efetividade e a segurança no uso dos medicamentos, respectivamente. Do total de PRM identificados, 82,2\% eram evitáveis e $63,7 \%$ eram manifestados. Destes, $85,5 \%$ eram causados por apenas um medicamento, o restante deveu-se às interações medicamentosas. Houve 163 medicamentos envolvidos com PRMs, a maioria pertencente aos sistemas: cardiovascular $(30,7 \%)$, sendo o captopril responsável pela ocorrência de $11,0 \%$ do total e; nervoso $(33,1 \%)$, sendo o clonazepam responsável por 3,7\% do total.

Das 114 intervenções registradas, $62,8 \%$ foram repassadas verbalmente e $71,1 \%$ foram destinadas aos pacientes. Houve situações em que não foi realizada qualquer intervenção como, por exemplo, quando o paciente não retornou ao serviço. Além disso, determinou-se que não seriam realizadas intervenções em situações cujos benefícios do tratamento superassem os riscos (PRM identificado). Houve a aceitação de 79\% das intervenções realizadas pelo farmacêutico destinadas ao paciente, cuidador ou profissional de saúde, sendo que em $71,7 \%$ destes casos, a aceitação foi total e nas demais a aceitação foi parcial. Considerou-se aceitação parcial quando, por exemplo, a intervenção sugeria a substituição do medicamento devido a este ser causa do PRM identificado, mas o prescritor considerou melhor opção a retirada da medicação, sem substituí-la. Dos 90 resultados conhecidos, 47,8\% levaram à estabilidade do caso, evitando o progresso do problema de saúde, e 31,1\% levaram à melhora.

Dos 58 pacientes acompanhados, 54 possuíam necessidade relacionada ao paciente (NRP), que totalizaram 151, média de 2,6 $( \pm 2,60)$ necessidades por paciente. A maioria dos pacientes buscava informações adicionais sobre a terapia farmacológica utilizada, como pode ser observado na Tabela 3. A orientação foi realizada, principalmente, de forma verbal em 71,5\% dos casos e $84,8 \%$ das necessidades foram supridas.

\section{Discussão}

Pôde-se observar um elevado uso de medicamentos por parte dos pacientes assistidos pelo serviço quando comparado ao perfil farmacológico dos pacientes incluídos nos estudos de Dall'Agnol et al. ${ }^{18}$ e Correr et al. ${ }^{19}$ cujo valor médio foi de 3,6 e 3,0 medicamentos por paciente, respectivamente. A idade avançada e a utilização de elevado número de medicamentos podem ser considera- 
Tabela 2. Frequência e classificação dos Problemas Relacionados aos Medicamentos, de acordo com o Segundo Consenso de Granada ${ }^{3}$, identificados nos pacientes atendidos no Serviço de Atenção Farmacêutica.

\begin{tabular}{lcc}
\hline \multicolumn{1}{c}{ Classificação do PRM } & n & $\%$ \\
\hline $\begin{array}{l}\text { PRM 1 (não utiliza o medicamento } \\
\text { que necessita) }\end{array}$ & 27 & 17,3 \\
$\begin{array}{l}\text { PRM 2 (utiliza um medicamento } \\
\text { que não necessita) }\end{array}$ & 3 & 1,9 \\
$\begin{array}{l}\text { PRM 3 (medicamento não é efetivo } \\
\text { para o paciente) }\end{array}$ & 11 & 7,0 \\
$\begin{array}{l}\text { PRM 4 (medicamento com dose } \\
\text { menor que a necessária) }\end{array}$ & 55 & 35,0 \\
$\begin{array}{l}\text { PRM 5 (medicamento provoca } \\
\text { reações adversas) }\end{array}$ & 36 & 22,9 \\
$\begin{array}{l}\text { PRM 6 (medicamento com dose } \\
\text { maior que a necessária) }\end{array}$ & 25 & 15,9 \\
\begin{tabular}{l} 
Total \\
\hline
\end{tabular} & 157 & 100,0 \\
\hline
\end{tabular}

Tabela 3. Frequência e classificação das Necessidades Relacionadas ao Paciente (NRP) identificadas entre os pacientes atendidos no Serviço de Atenção Farmacêutica.

\begin{tabular}{lcr}
\hline \multicolumn{1}{c}{ Classificação da NRP } & n & \multicolumn{1}{c}{$\%$} \\
\hline Informação sobre a doença & 11 & 7,3 \\
Cuidados com a alimentação & 22 & 14,6 \\
Incentivo a prática de exercícios & 6 & 4,0 \\
físicos & & \\
Monitoramento & 29 & 19,2 \\
Encaminhamento a profissionais de & 17 & 11,3 \\
saúde & & \\
Automedicação responsável & 6 & 4,0 \\
$\begin{array}{l}\text { Informação sobre a terapia } \\
\text { farmacológica }\end{array}$ & 46 & 30,4 \\
Outras terapias não-farmacológicas & 14 & 9,2 \\
Total & 151 & 100,0 \\
\hline
\end{tabular}

dos como fatores condicionantes para o número expressivo de PRM identificado, assim como afirmado por Baena et al. ${ }^{20}$. Além disso, pelo fato do SAF localizar-se em uma clínica multidisciplinar, os pacientes atendidos nesse serviço eram encaminhados, muitas vezes, por outros profissionais de saúde que, ao perceberem o número elevado de medicamentos usado pelo paciente, sentiam a necessidade de conduzi-los para uma análise dessa situação.

Em relação às classes farmacológicas mais envolvidas com problemas identificados nos pacientes prevaleceram os medicamentos que atuam nos sistemas nervoso e cardiovascular, cujo resultado assemelha-se ao descrito na literatura ${ }^{19,21}$. Castro et al..$^{22}$ identificaram em pacientes hipertensos, 66 problemas relacionados ao uso de medicamentos anti-hipertensivos, principalmente, referentes à segurança e à efetividade, assim como no presente estudo.

Segundo a classificação proposta pelo Segundo Consenso de Granada ${ }^{3}$ os PRM mais prevalentes neste estudo foram os 4 e 5. O PRM 4 consiste na utilização do medicamento obtendo-se uma efetividade inferior à desejada ${ }^{3}$ podendo ocorrer devido a interações entre o medicamento e a alimentação ou outro medicamento. Neste caso, o captopril foi o principal envolvido neste tipo de PRM pelo fato de ser usado com grande frequência concomitante à alimentação. Nesta situação as intervenções ocorreram para ajustar o horário de administração do medicamento com o objetivo de aumentar a efetividade do tratamento farmacológico ${ }^{23}$. Corroborando com os resultados encontrados no presente estudo, Dall'Agnol et al. ${ }^{18}$ e Baena et al. ${ }^{20}$ identificaram PRM relacionados à efetividade do medicamento, respectivamente em $66,7 \%$ e $62,7 \%$ dos casos estudados.

No presente estudo também houve a ocorrência de PRM 5, o qual diz respeito a inseguridade qualitativa do medicamento ${ }^{3}$ como, por exemplo, no caso de surgimento de reações adversas. Situação bastante frequente, neste estudo, entre os medicamentos que agem no sistema nervoso central, como os benzodiazepínicos, e no sistema cardiovascular, como os inibidores da Enzima Conversora de Angiotensina (ECA). Os benzodiazepínicos, como o bromazepam e o diazepam, foram responsáveis pela ocorrência de prejuízos da atividade motora e sonolência, enquanto os medicamentos inibidores da ECA, como o captopril, causaram, principalmente, tosse $\sec ^{23}$. Resultados semelhantes foram encontrados por Piá ${ }^{4}$, com aproximadamente 20,5\% dos casos de PRM tendo sido associados ao aparecimento de efeitos adversos.

No presente estudo, as interações medicamentosas foram responsáveis por $14,5 \%$ dos PRMs estando relacionadas, na maioria dos casos, à efetividade do tratamento, representadas, principalmente, pelo captopril e sua interação com alimentos, e à segurança, representadas por medicamentos atuantes no sistema nervoso central, como a interação entre paroxetina e risperidona, assim como a imipramina e o ácido valpróico $^{23}$. Correr et al. ${ }^{19}$ encontraram que a maioria das interações medicamentosas $(66 \%)$ rela- 
cionava-se ao comprometimento da efetividade da terapêutica, sendo, principalmente, entre captopril e alimento.

Quanto à possibilidade de se evitar problemas advindos do uso de medicamentos, Netto et al. ${ }^{24}$ também encontraram, ao analisar um serviço de emergência, no mesmo município onde foi realizado este estudo, quantidade elevada de PRM passível de ser evitado (72,7\%). Fato este importante quando se analisa o custo econômico devido aos atendimentos médicos causados por problemas que podem ser evitados a partir de uma assistência primária em saúde efetiva e de qualidade. Baena et al. ${ }^{1}$ destacam que cerca de $30 \%$ dos atendimentos hospitalares ocorrem devido a problemas que estão relacionados ao uso de medicamentos.

Cabe neste momento ressaltar a necessidade da atuação efetiva dos profissionais de saúde, especialmente farmacêuticos, envolvidos com os pacientes e seus medicamentos com vistas à redução de custos com a farmacoterapia ${ }^{25}$. Procedimentos aplicáveis à prática na farmácia comunitária, como a orientação sobre a forma de administração, intervalo e dosagem, são capazes de prevenir diversos problemas envolvidos com medicamentos.

Desta forma, as intervenções farmacêuticas para prevenção e resolução de PRM demonstraram ser bastante efetivas na resolução desses problemas, sejam elas destinadas diretamente ao paciente ou a outro profissional de saúde ${ }^{26}$. Destaca-se que, faz-se necessário o contato com o médico somente nos casos em que há necessidade de modificações no regime terapêutico de medicamentos que exigem prescrição ${ }^{7}$. Para Gérvas et al. ${ }^{7}$ a identificação de problemas relacionados a medicamentos torna-se mais fácil para os farmacêuticos por alguns motivos como, por exemplo, a maior acessibilidade geográfica e temporal, motivada pela existência de farmácias na maioria das regiões do país onde se pode conversar com um farmacêutico, na maioria das vezes, sem a necessidade prévia de agendamento, ao contrário do que ocorre na consulta médica.

Além disso, o elevado número de NRP identificado neste estudo revela a carência de informação dos pacientes na atenção primária em saúde. A NRP é uma necessidade identificada pelo paciente ou pela equipe de saúde, relacionada com a falta ou a deficiência de informação que reflete na compreensão desta por parte do paciente. Desta forma, estas necessidades podem incluir orientações sobre o problema de saúde, a terapêutica e o sistema de saúde.
Baena et al. ${ }^{1}$ afirmam que cada vez mais se faz necessário a implantação do acompanhamento farmacoterapêutico na atenção primária, a fim de obter o máximo benefício das medicações e estimular o desenvolvimento de políticas de saúde de caráter integral.

Para isto, a criação de equipes de saúde multidisciplinares e a necessidade de colaboração entre profissionais de saúde parecem ser primordiais para que as ações de saúde tenham a maior possibilidade de êxito, facilitando a atuação de um farmacêutico que trabalhe conjuntamente para a melhor qualidade da assistência ${ }^{1}$. No presente estudo, ficou evidente a inserção do Serviço de Atenção Farmacêutica na equipe multiprofissional de saúde quando do encaminhamento dos pacientes para outros profissionais de saúde ou mesmo destes para o SAF.

Ressalta-se que o Serviço de Atenção farmacêutica, aqui descrito, representa uma nova forma de atuação do farmacêutico que pode ser desenvolvida junto à equipe do Núcleo de Apoio à Saúde da Família (NASF), quando este profissional puder ser inserido nesse contexto. Segundo Araújo et al. ${ }^{27}$, a Atenção Farmacêutica pode ser considerada como uma especialidade da tecnologia do uso do medicamento, privativa do farmacêutico, porém ainda incipiente no Sistema Público de Saúde. Neste, o profissional farmacêutico poderá prestar atendimento a grupos especiais de pacientes, como diabéticos e hipertensos ou de acordo com a necessidade da região de sua atuação. Através da atenção farmacêutica, será possível proporcionar o uso racional de medicamentos, consequentemente, maior qualidade de vida aos pacientes, além de interagir com a equipe multiprofissional, o que se mostrou extremamente necessário neste estudo.

\section{Conclusões}

Durante a realização deste estudo foram identificados, principalmente, problemas relacionados aos medicamentos relacionados à efetividade e à segurança, sendo a maioria evitáveis e manifestados. As intervenções realizadas pelos farmacêuticos, na maioria dos casos, destinaram-se aos próprios pacientes e foram comunicadas verbalmente, contribuindo para o alcance de melhores resultados na terapia farmacológica e, por outro lado, para a estabilidade do problema de saúde.

Além desses indicadores, foram percebidas, principalmente, necessidades do paciente sobre informações acerca da terapia farmacológica, que 


\section{Colaboradores}

GM Alano, TS Corrêa e D Galato participaram igualmente de todas as etapas de elaboração do artigo.

\section{Referências}

1. Baena MI, Olmos JM, Faus MJ, Martínez FM. Seguimiento farmacoterapéutico integral de pacientes em el sistema sanitário. Rev Pharm Care Esp 2002; 4(5):325-332.

2. Panel de Consenso. Tercer Consenso de Granada sobre Problemas Relacionados com Medicamentos (PRM). Ars Pharm 2007; 48(1):5-17.

3. Panel de Consenso. Segundo Consenso de Granada sobre Problemas Relacionados con Medicamentos. Ars Pharm 2002; 43(3-4):175-184.

4. Piá CP. Identificación y resolución de problemas relacionados con los medicamentos: experiencia en un centro de salud. Rev Pharm Care Esp 2002; 4(5):300-313

5. Ivama AM, Noblat L, Castro MS, Oliveira NVBV, Jaramillo NM, Rech N. Consenso Brasileiro de Atenção Farmacêutica: proposta. Brasília: Organização Pan-Americana de Saúde (OPAS); 2002.

6. Cipolle RJ, Strand LM, Morley PC. El Ejercicio de la Atención Farmacéutica. Madrid: McGraw-Hill; 1998.

7. Gérvas J, Toledo FA, Erayalar MT. Limitaciones clave de la Atención Farmacéutica como respuesta a los problemas relacionados con los medicamentos. Pharm Care Esp 2003; 5(1):55-58.

8. Mota DM. Avaliação farmacoeconômica: instrumentos de medida dos benefícios na atenção farmacêutica. Acta Farm Bonaerense 2003; 22(1):73-80.

9. Hernández DS, Castro MMS, Dáder MJF. Programa Dáder. Método Dáder. Guía de seguimento farmacoterapéutico. Granada: Universidade de Granada; 2007

10. Vinholes ER, Alano GM, Galato D. A percepção da comunidade sobre atuação do Serviço de Atenção Farmacêutica em ações de Educação em Saúde relacionadas a Promoção do Uso Racional de Medicamentos. Saúde Soc 2009; 18(2):302-312.

11. Galato D, Alano GM, Valgas C. Implantação de um Serviço de Atenção Farmacêutica (SAF) em um ambiente multiprofissional de atendimento ambulatorial (relatório técnico). Brasília: Conselho Nacional de Desenvolvimento Científico e Tecnológico ( $\mathrm{CNPq})$; 2008.

12. Associação Brasileira de Empresas de Pesquisa (ABEP). Critério de Classificação Econômica Brasil. [site na Internet]. [acessado 209 mar 15]. Disponível em: http://www.abep.org/codigosguias/Criterio_ Brasil_2008.pdf 
13. Who Collaborating Centre for Drug Statistics Methodology. Atc/ddd Index 2008. [site na Internet]. [acessado 2008 abr. 10]. Disponível em: http:// www.whocc.no/atcddd/.

14. Otero MJ, Bajo A, Maderuelo JA, Domínguez-Gil A. Evitabilidad de los acontecimientos adversos inducidos por medicamentos detectados en un Servicio de Urgencias. Rev Clin Esp 1999; 199:796-805.

15. EpiData Data Entry. Data managemente and basic statistical analysis system [programa de computador]. Versão 3.0. Odense: EpiData Association; 2002.

16. Epi Info [programa de computador]. Versão 3.32. Atlanta: CDC Centers for Disease Control and Prevention; 2005.

17. Brasil. Resolução RE Nº 196 de 10 de outubro de 1996. Estabelece os requisitos para realização de pesquisa clínica de produtos para saúde utilizando seres humanos. Diário Oficial da União 1996; 16 out.

18. Dall'Agnol RSA, Albring DV, Castro MS, Heineck I. Problemas Relacionados com Medicamentos em Serviço de Emergência de Hospital Universitário do Sul do Brasil. Estudo Piloto. Acta Farm Bonaerense 2004; 23(4):540-545.

19. Correr CJ, Pontarolo R, Ferreira LC, Baptistão SAM. Riscos de problemas relacionados com medicamentos em pacientes de uma instituição geriátrica. Rev Bras Cienc Farm 2007; 43(1):55-62.

20. Baena MI, Fajardo P, Luque FM, Marin R, Arcos A, Zarzuelo A, Jiménez J, Faus MJ. Problemas relacionados con los medicamentos en usuarios de un servicio de urgencias hospitalario: resultados de la validación de un cuestionario. Pharm Care Esp 2001; 3(5):345-357.

21. González JAB, Galán TA. Detección y resolución de problemas relacionados con los medicamentos en la farmacia comunitaria: una aproximación. Pharm Care Esp 1999; 1(2):113-122.

22. Castro MS, Chemello C, Pilger D, Junges F, Bohnen L, Zimmerman LM, MA Paulino, Jacobs U, Ferreira MBC, Fuchs FD. Contribuição da atenção farmacêutica no tratamento de pacientes hipertensos. Rev Bras Hipertens 2006; 13(3):198-202.

23. Lacy CF, Armstrong LL, Goldman MP Lance LL. Drug information handbook. Ohio: Lexi-Comp; 2007.

24. Netto, ASM, Melo FB, Silva WB. Frecuencia de problemas relacionados con los medicamentos en pacientes que visitaron el servicio de urgencia de un hospital regional. Seguimiento Farmacoterapéutico 2005; 3(4):213-224.

25. Vieira FS. Possibilidades de contribuição do farmacêutico para a promoção da saúde. Cien Saude Colet 2007; 12(1):213-220.

26. Lyra Júnior DP, Marcellini PS, Péla IR. Effect of pharmaceutical care intervention on blood pressure of elderly outpatients with hypertension. Rev Bras Cienc Farm 2008; 44(3):451-457.

27. Araújo ALA, Pereira LRL, Ueta JM, Freitas O. Perfil da assistência farmacêutica na atenção primária do sistema único de saúde. Cien Saude Colet 2008; 13(Supl.):611-617.

Apresentado em 10/05/2010

Aprovado em 20/07/2010

Versão final apresentada em 20/08/2010 